\title{
Comportamento violento e disfunção cerebral: estudo de homicidas no Rio de Janeiro*
}

\section{Violent behavior and brain dysfunction: study of murderers in Rio de Janeiro}

\author{
Flavio Jozef, Jorge Adelino R da Silva, Sandra Greenhalgh**, Maria Esther D Leite** e Vania H Ferreira***
}

Serviço de Psiquiatria Forense do Instituto de Psiquiatria da Universidade Federal do Rio de Janeiro

Resumo Objetivos: Estudar a correlação entre disfunção cerebral e psicopatia em homicidas.

Métodos: Foram separados em dois grupos (psicopatas e não-psicopatas) 29 homicidas "normais" (não-psicóticos), detidos em uma delegacia policial e escolhidos aleatoriamente, com base no HARE PCL-R (escala de avaliação de psicopatia). Ambos os grupos foram submetidos a testagem neuropsicológica, sendo empregados testes voltados para atividade em lobo frontal (Trail Making Test A e B, e subtestes do WAIS [Mosaico, Semelhanças e Símbolos Numéricos]).

Resultados: Dos homicidas, 15 foram considerados psicopatas e 14, não-psicopatas. O subteste Mosaico, do WAIS, constituiu-se em discriminador entre os dois grupos pela presença significativa de resultados negativos em não-psicopatas $\left(\chi^{2}=5,37 ;\right.$ G.L. $\left.=1 ; \mathrm{P}<0,05\right)$. Como a prevalência de diagnósticos de dependência/abuso de álcool/drogas foi maior entre os psicopatas, conclui-se que esse fator não influiu significativamente na melhor performance neuropsicológica desse grupo.

Conclusões: Há evidências de correlação entre disfunção cerebral frontal e comportamento homicida em nãopsicopatas. Sugere-se o emprego sistemático do PCL-R em populações forenses violentas, com o objetivo de atingir uma melhor depuração dos grupos estudados.

Descritores Homicídio. Comportamento violento. Psicopatia.

Abstract Objectives: The aim of the study was to investigate the association between psychopathy and cerebral dysfunction in a population of murderers.

Methods: A random sample of 29 "normal" (non-psychotic) murderers detained in a police station were evaluated and classified into psychopaths $(n=15)$ and non-psychopaths $(n=14)$ according to the HARE PCL-R. All individuals in the sample were submitted to neuropsychological tests (Trail Making Test A and B, and WAIS subtests [Block Design, Similarities and Digit Symbol]).

Results: The WAIS subtest Block Design was a discriminator between the sample subgroups, with psychopaths scoring significantly better than non-psychopaths $\left(c^{2}=5.37\right.$; G.L. $\left.=1 ; p<0.05\right)$. As psychopaths were most commonly diagnosed with alcohol/illicit drugs addiction/abuse than non-psychopaths, this factor does not seem to account for the better neuropsychological performance of non-psychopaths.

Conclusions: There is evidence that frontal lobe dysfunction is implied in homicidal behavior among nonpsychopaths. A better psychiatric evaluation of murderers and the routine use of HARE PCL-R as a clinical and research tool are recommended.

Keywords Homicide. Violent behavior. Psychopathy. 


\section{Introdução}

Violência e crime são objetos de preocupação em todo o mundo. Estatísticas no Brasil apontam para um alto índice de homicídios, o que certamente reflete uma sociedade extremamente violenta.

Por outro lado, gradativamente vem-se avolumando um corpo de conhecimento relativo a alterações no funcionamento cerebral de indivíduos violentos. A correlação entre estruturas cerebrais específicas e o comportamento agressivo em mamíferos e primatas antropóides também vem sendo amplamente estudada. ${ }^{1}$

Essa correlação, pelo menos no que diz respeito a um subgrupo de indivíduos agressivos de forma contumaz, tem sido estabelecida por pesquisas, de forma mais intensa, no decorrer das últimas três décadas, ${ }^{1-16}$ sem ignorar, porém, a grande causalidade envolvida na questão da violência e do comportamento violento. Dentro dessa visão multifatorial, desenvolve-se e articula-se uma vertente que podemos chamar de cerebral. Definise comportamento violento como "uso intencional de força ou ação física contra uma pessoa, seja como coação ou como um fim em si, provocando dano físico ou moral na vítima", sendo que, necessariamente, esse comportamento será criminoso.

Porém, freqüentemente tem sido apontada, como um dos empecilhos metodológicos e fatores de distorção nesses estudos, a heterogeneidade das amostras estudadas e apreendidas, não raro, por "critérios de oportunidade", como "indivíduos encaminhados para perícia psiquiátrico-forense ao longo do tempo", assim como comportamentos violentos em questão, por vezes imprecisamente definidos. ${ }^{17}$

Nesse sentido, estudos que apresentem um melhor enquadramento do comportamento violento estudado, como é o caso dos estudos com homicidas, apresentam vantagens. Homicídio é universalmente considerado o crime capital, trazendo importante carga de violência implícita. No presente estudo, 29 homicidas detidos em delegacia policial, no Rio de Janeiro, foram investigados. Desse total, puderam-se classificar nove homicídios atuais, (isto é, que motivaram a detenção atual) como "instrumentais" (relacionados ao cometimento de "outros crimes"), e 18 como "reativos" (vinculados a discussões ou disputas).*

No tocante ao estudo de populações forenses heterogêneas, como é, evidentemente, o caso de "homicidas", considerou-se que instrumentos de avaliação de personalidade como o HARE PCL- $\mathrm{R}^{18}$ representam necessidade e também importante avanço, na medida em que esse instrumento foi desenvolvido, especificamente, para emprego em tais populações (forenses), ao contrário de outros, como o MMPI, permitindo efetuar uma “depuração" eficaz nesses grupos.

$\mathrm{Na}$ avaliação do funcionamento cerebral em populações violentas, têm sido empregados neuroimagem (TCC, RM, PET), EEG, avaliação neurológica minuciosa e testagem neuropsicológica. Para Lewis, ${ }^{19}$ inclusive, a testagem neuropsicológica é dos mais precisos instrumentos, especialmente se comparado ao exame neurológico tradicional. $\mathrm{Ou}-$ tra vantagem da avaliação neuropsicológica é a sua flexibi- lidade, a possibilidade de composição de baterias específicas, adequadas à avaliação de determinadas populações/regiões cerebrais.

\section{Disfunção em lobos frontais e hemisfério cerebral esquerdo}

Uma questão controversa diz respeito à região cerebral onde se localizaram as disfunções constatadas em populações violentas. Inicialmente, talvez em função do peso da epilepsia de lobo temporal (ELT) e sua correspondente em comportamento violento, houve uma tendência a associar violência a disfunção dos lobos temporais e sistema límbico. ${ }^{6}$ Em 1974, em revisão da literatura, Goldstein ${ }^{15}$ concluiu pela fragilidade dessa relação. Posteriormente, inclusive, foi indicada uma maior associação entre crime violento e epilepsia generalizada, comparando-se com a ELT. ${ }^{11}$

Em fase posterior, Spellacy, ${ }^{10}$ em 1977, e Yeudall, ${ }^{14} \mathrm{em}$ 1982, preferiram falar em "perfis neuropsicológicos anormais" ou "comprometimento na função cerebral", respectivamente, referindo-se assim às alterações detectadas nas populações violentas que estudaram, evidenciando uma tendência a apontar a presença de disfunção cerebral difusa em indivíduos violentos.

Na década de 90 os estudos tenderam, de forma crescente, a se voltar para lobo frontal e hemisfério cerebral esquerdo e para a correlação de suas disfunções com comportamento violento.

A atividade dos lobos frontais envolve o controle e a regulação do comportamento, bem como a aptidão para formar e manter um plano de ação ou, ainda, alterá-lo de forma adequada quando já em execução. Pontius \& Yudowitz, ${ }^{8}$ em 1980, postularam que criminosos violentos sofreriam de comprometimento na capacidade de alterar ações já iniciadas, o que os levaria a situações de risco no tocante à violência. No estudo que efetuaram com "adultos jovens criminosos", empregando o Trail Making Test-B (TMT-B), encontraram indicações de disfunção frontal em $33 \%$ dos indivíduos. Volkow \& Tancredi, ${ }^{13}$ em 1987, avaliaram a atividade cerebral de quatro "pacientes psiquiátricos violentos", empregando o PET, TCC e EEG; apontando a presença de disfunção em córtex frontal em dois e temporal em todos. Yeudall ${ }^{14}$ referiu sinais de comprometimento em região fronto-temporal anterior de hemisfério cerebral nãodominante, chamando a atenção para as marcantes diferenças entre delinqüentes e grupo-controle. Raine et $\mathrm{al}^{9}$ descreveram, em 1996, a presença de hipometabolismo em lobo frontal nos "homicidas insanos" que estudaram com emprego do PET. Lishman, ${ }^{20}$ examinando pacientes com história de TCE e lesões cerebrais, encontrou comportamento criminal ou anormalidades sexuais apenas associados a lesões frontais. Heinrichs, ${ }^{4}$ em 1989, examinando pacientes neuropsiquiátricos crônicos com comportamento violento, com o emprego de TCC, referiu a presença de lesões focais frontais.

Blake et $\mathrm{al}^{2}{ }^{2}$ em 1995, estudaram 31 homicidas empregando EEG, exame neurológico, MRI, TCC e testagem neuropsicológica, relatando a presença de disfunção frontal em $64,5 \%$ dos indivíduos. Sreenivasan et al, ${ }^{21}$ em 1997, estudando pacientes psiquiátri- 
cos "violentos de forma criminosa", vis a vis não-violentos, com emprego de testagem neuropsicológica e do HARE PCL-R, concluíram que se constituíam em importantes preditores de recidividade violenta a "insuficiência de freios morais", avaliada pelo PCL-R, bem como a "inflexibilidade cognitiva", além de "função espacial inadequada”, sendo, essas últimas, deficiências neurológicas ligadas a patologia pré-frontal (lateral-dorso-frontal) e avaliadas pelo TMT-B, Mosaico e Wisconsin Card Sorting Test.

Assim, toda uma série recente de estudos tem correlacionado comportamento violento grave a alterações neuropsiquiátricas, especialmente no que se refere aos lobos frontais e também ao hemisfério cerebral esquerdo.

\section{Psicopatia e HARE PCL-R}

Para Hare, ${ }^{22}$ uma deficiência de muitos estudos de populações criminais consistiria em não avaliar e valorizar devidamente a presença de psicopatas, situação que se refletiria, inclusive, na DSM-III, DSM-III-R e DSM-IV, levando esses estudos a distorções. O conceito de APD (Anti-social Personality Disorder), encontrado nessas classificações, incidiria circularidade com "populações criminosas", por se definir basicamente pelo comportamento criminal, além de não apreender o que mais caracterizaria o psicopata, ou seja, seu núcleo de características afetivas e interpessoais. O HARE PCL-R, escala de avaliação de psicopatia desenvolvida por HARE e seu grupo, é fruto de 25 anos de pesquisa e tem sido amplamente empregada em estudos forenses. Ela reflete a presença do constructo psicopata de forma válida e fidedigna, significando uma retomada da tradição clínica, por se ancorar nas clássicas descrições de autores como Cleckley, ${ }^{23}$ bem como por revalorizar a intuição e o sentimento clínicos para diagnóstico. Essa escala foi desenvolvida a partir das 16 características que definem o perfil do psicopata, de Cleckley, objetivando operacionalizar o constructo psicopatia. Ela consta de 20 itens, que são pontuados de 0 a 2, conforme a adaptação do indivíduo a determinado traço. Para HARE, psicopatia se constituiria em um distúrbio socialmente devastador, definido por uma constelação de características afetivas, interpessoais e comportamentais, incluindo egocentrismo, impulsividade, ausência de empatia, culpa ou remorso, mentira patológica e persistente violação de normas e expectativas. Em última análise, psicopatas seriam predadores intra-específicos, que empregariam quaisquer meios para satisfação de suas próprias necessidades egoístas, inclusive violência.

\section{Estudo com homicidas no Rio de Janeiro}

\section{Objetivos}

Tendo em vista a relevância do problema da criminalidade violenta e do homicídio, particularmente no Brasil, onde ambos atingem incidência de alarmantes proporções, ${ }^{17,24}$ visou-se realizar uma primeira abordagem do problema. Anteriormente, Sil$\mathrm{va}^{25}$ estudou delinqüentes juvenis no Rio de Janeiro, avaliando a incidência de patologia psiquiátrica. $\mathrm{O}$ estudo com homicidas visava verificar a incidência de alterações neuropsicológicas e de personalidade (psicopatia) em um grupo representativo dessa população. Enfatizou-se o fato de se tratar de um grupo de "homicidas normais", ou seja, isentos, em princípio, de patologia mental: o sistema penal, por imperativo legal, transfere os criminosos doentes mentais para o Manicômio Judiciário ou para o Hospital Psiquiátrico Penitenciário.

\section{Métodos}

O grupo de homicidas foi escolhido ao acaso, por meio do exame de todos os detidos incursos no artigo 121 do CPB (homicídio e tentativa de homicídio), que se encontravam detidos em uma delegacia policial do Rio de Janeiro, em um dado momento, escolhido ao acaso. Dos 34 indivíduos que se encaixavam nos critérios de inclusão, 5 recusaram-se a participar da pesquisa e 2 foram transferidos para o sistema penitenciário. Nos 20 dias posteriores foram incluídos outros 2 indivíduos, então transferidos para a delegacia, que preenchiam os requisitos e concordaram em participar. Dos 29 presos, 2 recusaram-se a realizar a testagem neuropsicológica, e dos restantes, 3 resultados foram desprezados devido à avaliação de falta de cooperação.

\section{Instrumentos e procedimentos}

Os indivíduos foram submetidos, após seu consentimento informado, à aplicação do HARE PCL-R e à testagem neuropsicológica (Trail Making Test A e B, e subtestes do WAIS [Semelhanças, Mosaico e Símbolos Numéricos]). Mosaico ${ }^{26}$ é um subteste considerado sensível para lesões em hemisfério cerebral esquerdo, lobo frontal ou ainda lesões extensas em hemisfério cerebral direito. Ele avalia função espacial, organização perceptual, capacidade de análise e síntese. Consiste na arrumação de pequenos cubos vermelhos e brancos, buscando reproduzir desenhos apresentados em cartões impressos. O Trail Making

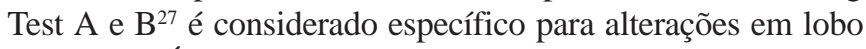
frontal. ${ }^{2,8,14}$ É um teste de habilidade cognitiva simples, de lápis e papel. Consiste em ligar círculos numerados o mais rápido possível, com um lápis, sem tirá-lo do papel. A primeira folha tem números crescentes; a segunda, números e letras alternados. O tempo é cronometrado e comparado posteriormente.

Foi empregada anamnese semipadronizada adaptada de Sil$\mathrm{va},{ }^{25}$ de forma a permitir aplicação do HARE PCL-R. Esse consiste de 20 itens, avaliados e pontuados de 0 a 2 , para cada indivíduo, com base em entrevista pessoal e informações colaterais (de arquivo). Os critérios para a pontuação são explicitados em manual próprio ${ }^{18}$ e implicam no conhecimento do trabalho de Robert Hare e seu grupo, bem como da literatura sobre psicopatia. Adicionalmente, foram examinados por pesquisador membro da equipe (VHF), os processos criminais dos presos, instaurados em varas criminais do foro do Rio de Janeiro, por incluírem informações colaterais adicionais, como circunstâncias dos crimes e passado criminal dos indivíduos, imprescindíveis para aplicação do PCL-R. ${ }^{18}$ Foi empregado o ponto de corte de 25 para o PCL-R, o mais utilizado na atualidade, segundo $\operatorname{Serin}^{28} \mathrm{e}$ Rice et al. ${ }^{29}$ A testagem neuropsicológica foi aplicada por psicóloga membro da equipe (MEDL), de forma cega em relação ao PCL-R e anamnese psiquiátrica, que foram aplicados por outro 
pesquisador $(\mathrm{FJ})$ e vice-versa. $\mathrm{O}$ não emprego de uma bateria neuropsicológica fixa, como Halstead-Reitan, Luria-Nebraska ou o WAIS completo, deveu-se à recomendação de que se adapte a bateria às finalidades do exame em questão. Ademais, tal aplicação demandaria de 6 a 8 horas, o que seria impraticável, face às limitações do ambiente de pesquisa.

Nos subtestes do WAIS (Mosaico, Semelhanças e Símbolos Numéricos) foram considerados anormais os resultados com dois ou mais desvios-padrão abaixo da média, de forma coerente com as recomendações da literatura. Com relação ao Trail Making Test A e B, foi levada em conta a presença de erros no Trail Making, bem como o tempo A menos tempo B maior do que 1 minuto, índice desenvolvido para essa população.

\section{Resultados}

As idades variaram de 19 a 58 anos. A média foi de 31,1 anos; $37,9 \%$ se concentraram na faixa de 18 a 25 anos. A maioria $(75,8 \%)$ tinha primeiro grau incompleto; 4 , primeiro grau completo; 3 , segundo grau completo. Não havia analfabetos, nem indivíduos com terceiro grau, devido ao fato de que presos com nível superior são detidos em outro local.

\section{Diagnósticos psiquiátricos}

Ao exame dos indivíduos, por meio de anamnese semipadronizada, acrescido dos resultados da aplicação do PCL-R e testagem neuropsicológica, foram encontrados os seguintes diagnósticos psiquiátricos, conforme os critérios da

Tabela 1 - Distribuição dos diagnósticos do CID-10 em amostra de homicidas na cidade do Rio de Janeiro.

\begin{aligned} \hline $\mathrm{N} &$ Diagnósticos psiquiátricos na amostra \\ \hline 1 & sem diagnóstico \\ 2 & psicopata \\ 3 & sem diagnóstico \\ 4 & psicopata \\ 5 & psicopata \\ 6 & psicopata + uso nocivo de cannabis (F.12.1) \\ 7 & psicopata + uso de múltiplas drogas (F.19) (internação psiquiátrica \\ & prévia) \\ 8 & psicopata \\ 9 & sem diagnóstico \\ 10 & psicopata + uso nocivo de álcool (F.10.1) \\ 11 & uso nocivo de álcool (F.10.1) \\ 12 & psicopata + uso nocivo de álcool (F.10.1) \\ 13 & psicopata + síndrome de dependência à cocaína (F.14.21) (divs. \\ & Internações psiq.) \\ 14 & psicopata + traços paranóides \\ 15 & psicopata \\ 16 & psicopata + traços paranóides + Uso nocivo de cannabis (F.12.1) \\ 17 & sem diagnóstico \\ 18 & uso nocivo de álcool (F.10.1) \\ 19 & uso nocivo de cocaína (F.14.1) + traços paranóides \\ 20 & sem diagnóstico \\ 21 & psicopata + uso nocivo de álcool (F.10.1) \\ 22 & uso nocivo de cocaína (F.14.1) \\ 23 & transtorno de personalidade Borderline (F.60.31) \\ 24 & psicopata + uso de múltiplas drogas (F.19.1) + traços paranóides \\ 25 & (fez tratamento psiq.) \\ 26 & sem diagnóstico \\ 27 & retardo mental leve (F.70) \\ 28 & psicopata + uso nocivo de cocaína (F.14.1) + traços paranóides \\ & traços paranóides \\ & \\ & \\ & \\ & \end{aligned}

CID- $10,{ }^{30}$ e de Hare ${ }^{18}$ para psicopatia. Adicionalmente, foi dado destaque à presença de traços paranóides, por sua importância especificamente no estudo de homicidas ${ }^{2}$ (Tabela 1 ).

\section{Lista geral de diagnósticos}

O constructo psicopatia foi empregado conforme os critérios de HARE com o objetivo de se obter maior precisão no estudo de distúrbios de personalidade. Dos 27 indivíduos que realizaram a testagem neuropsicológica, 2 visivelmente não colaboraram, alegando dificuldade do exame, ou recusando-se a realizar as tarefas. Outro alegou incapacidade, por diminuição da acuidade visual. Assim, 24 resultados neuropsicológicos foram considerados. Desses, houve um indicação formal de retardo mental leve.

$\mathrm{Na}$ avaliação dos resultados do TMT A e B, foi levada em conta a discrepância entre os tempos B e A, bem como a presença de erros no TMT-B. Dos 24 indivíduos, 16 apresentaram anormalidade em um dos índices e 8, em ambos.

Já na avaliação dos resultados de Semelhanças, Mosaico e Símbolos Numéricos, dos 24 indivíduos, 15 apresentaram pelo menos um resultado anormal, 6 apresentaram dois e 1 apresentou resultado anormal nos três subtestes.

Tabela 2 - Resultados neuropsicológicos dividindo-se a amostra em psicopatas e não-psicopatas.

\begin{tabular}{|c|c|c|c|c|}
\hline \multicolumn{5}{|c|}{ Psicopatas ( $\mathrm{N}=11)$} \\
\hline \multicolumn{4}{|c|}{ WAIS } & TMT A e B \\
\hline Casos & Semelhanças & Mosaico & $\begin{array}{l}\text { Símbolos } \\
\text { numéricos }\end{array}$ & $\begin{array}{ll}\text { Erros } & \mathrm{T}_{\mathrm{b}}-\mathrm{T}_{\mathrm{a}}>1^{\prime}\end{array}$ \\
\hline
\end{tabular}

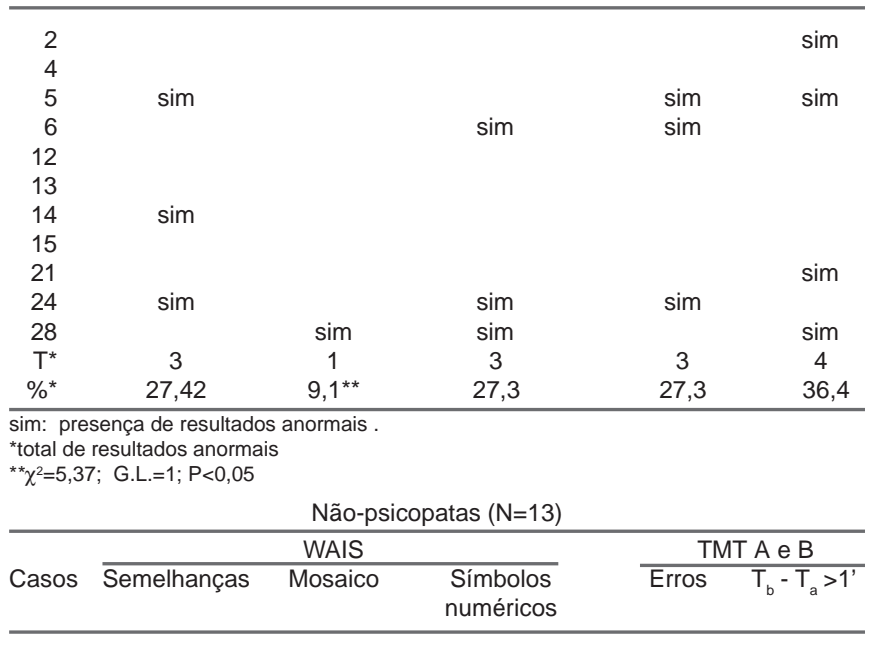

\begin{tabular}{|c|c|c|c|c|c|}
\hline 1 & $\operatorname{sim}$ & & & & $\operatorname{sim}$ \\
\hline 3 & & $\operatorname{sim}$ & & $\operatorname{sim}$ & $\operatorname{sim}$ \\
\hline 9 & & $\operatorname{sim}$ & & $\operatorname{sim}$ & $\operatorname{sim}$ \\
\hline 11 & & $\operatorname{sim}$ & $\operatorname{sim}$ & $\operatorname{sim}$ & $\operatorname{sim}$ \\
\hline 17 & $\operatorname{sim}$ & & $\operatorname{sim}$ & $\operatorname{sim}$ & $\operatorname{sim}$ \\
\hline 18 & & sim & & & sim \\
\hline 20 & & & & $\operatorname{sim}$ & $\operatorname{sim}$ \\
\hline 22 & & & & & \\
\hline 23 & $\operatorname{sim}$ & $\operatorname{sim}$ & $\operatorname{sim}$ & $\operatorname{sim}$ & $\operatorname{sim}$ \\
\hline 25 & & $\operatorname{sim}$ & & & \\
\hline 26 & & & & $\operatorname{sim}$ & \\
\hline 27 & $\operatorname{sim}$ & & $\operatorname{sim}$ & $\operatorname{sim}$ & \\
\hline 29 & $\operatorname{sim}$ & sim & & & $\operatorname{sim}$ \\
\hline $\mathrm{T}^{*}$ & 5 & 7 & 4 & 8 & 9 \\
\hline$\% *$ & 38,5 & $53,8^{* *}$ & 30,8 & 61,5 & 69,2 \\
\hline
\end{tabular}


Tendo por base os resultados do PCL-R, desdobrou-se o grupo original de 29 presos homicidas em 2 subgrupos: psicopatas (P), composto de 15 indivíduos $(51,72 \%)$, e não-psicopatas (NP), composto de 14 indivíduos, (48,28\%). Desses, 24 indivíduos $(\mathrm{P}=11 ; \mathrm{NP}=14)$ realizaram a testagem neuropsicológica e tiveram seus resultados avaliados (Tabela 2).

Verificou-se uma concentração de resultados deficitários em NP. Especificamente em relação ao TMT-B, de 24 índices anormais encontrados em toda a amostra, 7 se alocaram em $\mathrm{P}(\mathrm{N}=11)$ e 17 em NP ( $\mathrm{N}=13)$. A média foi de, respectivamente, 0,64 (DP 0,67) e 1,31 (DP 0,75) índices anormais por indivíduo. Tais resultados alcançaram significância estatística $(\mathrm{t}=2,29$; G.L. $=22 ; \mathrm{P}=0,032$ ).

Somando-se os resultados dos subtestes do WAIS, um total de 14 índices anormais foram encontrados em P (média de 1,27 [DP 1,27] por indivíduo) enquanto que 33, nos NP (média de 2,54 [DP 1,4] por indivíduo). Esses resultados são estatisticamente significativos $(\mathrm{t}=2,31 ; \mathrm{G} . \mathrm{L} .=22 ; \mathrm{P}=0,031)$. Foi empregado o teste do qui-quadrado na avaliação das variáveis categoriais. O teste T de Student foi empregado na avaliação de variáveis contínuas, na comparação de médias.

\section{Discussão}

A escolha de testes neuropsicológicos com sensibilidade para déficits no lobo frontal (LF) deveu-se a indicações da literatura. Por outro lado, os estudos relativos a psicopatas, no que diz respeito aos testes neuropsicológicos usuais, parecem poupálos de déficits em LF, hipótese já investigada anteriormente por Hare e seu grupo. ${ }^{31,32}$ Portanto, seria de se esperar que, com emprego da testagem neuropsicológica, o subgrupo P comportar-se-ia como um grupo controle "normal", afastando, ao mesmo tempo, um fator de confusão para o estudo da outra população violenta, (os NP) a saber, o "fator psicopatia".

Foram constatadas algumas outras divergências entre os subgrupos P e NP. O primeiro apresentou média de idade de 26 anos (DP 6,18), enquanto que NP apresentou média de 36,71 (DP 12,3), com uma diferença significativa ( $\mathrm{t}=3,00 ; \mathrm{G} . \mathrm{L} .=27 ; \mathrm{P}<0,01)$.

Essa tendência a uma maior pontuação no HARE PCL-R entre indivíduos mais jovens aparece quando se correlaciona idade e pontuação individual no PCL-R, verificando-se uma tendência à correlação negativa $(\mathrm{r}=-0,65 ; \mathrm{P}<0,01)$. Esse fato foi ilustrado graficamente (Figura).

A divisão do grupo de homicidas em P e NP, com emprego do PCL-R, permitiu também a caracterização desses 2 subgrupos de forma consistente, pelos resultados neuropsicológicos parcialmente divergentes para ambos.

No tocante a outros diagnósticos psiquiátricos na amostra (Tabela 1), houve importante presença de diagnósticos ligados a álcool e drogas. Do total de 29, 14 indivíduos $(48,27 \%)$ receberam algum diagnóstico ligado ao uso de álcool ou drogas. Porém P, que obteve melhores resultados neuropsicológicos, obteve também maior número desses diagnósticos, o que tem relevância: os piores resultados obtidos pelos NP não podem ser explicados como efeito de substâncias que comprometem o desempenho.

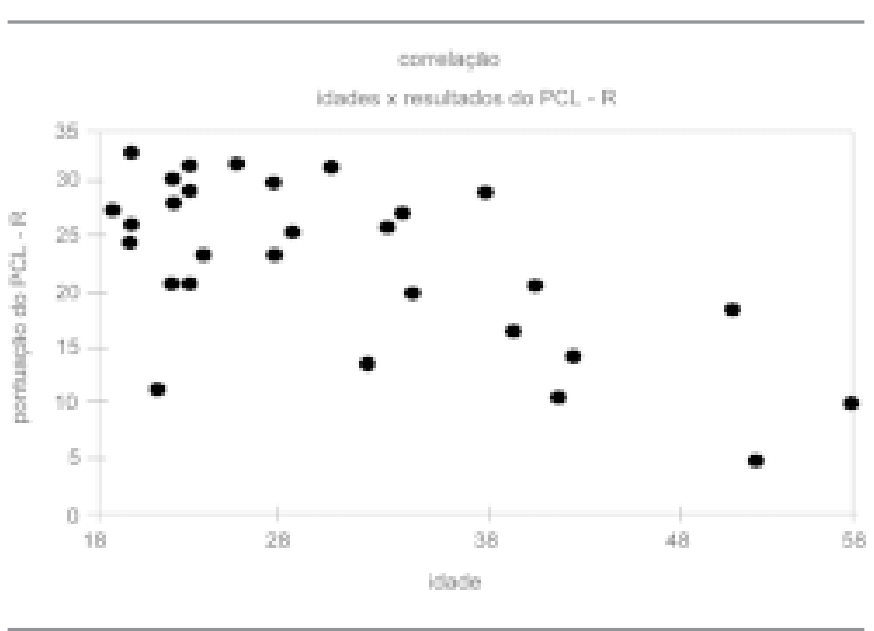

Figura - Correlação entre a idade dos homicidas e os escores no PCL-R

Quanto às características do crime cometido, os NP tenderam a cometer homicídios do tipo "reativo" ou "impulsivo", motivados ou desencadeados por raiva. ${ }^{33}$ Já os $\mathrm{P}$, tenderam a incidir nos homicídios "instrumentais", aqueles que ocorrem no âmbito de uma criminalidade mais ampla, com menor fator emocional envolvido (na disputa por pontos de drogas, em entreveros com a polícia, no decorrer, enfim, do cometimento de outros crimes), se bem que psicopatas também cometam homicídios reativos.

O subteste Mosaico, do WAIS, já foi apontado na literatura como tendo o poder de discriminar entre "pacientes psiquiátricos com alto e baixo índice de violência", 5 e como "preditor de recidividade em pacientes psiquiátricos violentos". ${ }^{21}$ No presente estudo, o subgrupo P obteve 90, $1 \%$ de resultados normais, enquanto os NP obtiveram apenas 46,2\% $\left(c^{2}=5,37\right.$; G.L. $=1 ; \mathrm{P}<0,05$ ). Isso parece confirmar a eficiência de Mosaico na discriminação de certos grupos violentos. É possível que tanto Mosaico quanto TMT-B, por detectarem déficits ligados a LF, por um lado "liberem" psicopatas, capturando outros grupos de indivíduos violentos.

Yeudall et al ${ }^{14}$ postularam que alterações em região límbico-orbital de LF levariam a explosões emocionais, por diminuição do autocontrole e aumento da impulsividade. Já alterações em região dorso-lateral de LF levariam a déficits na linguagem, na atenção, na organização de planos e intenções. Para Mega \& Cummings, ${ }^{34}$ o circuito lateral orbitofrontal, articulado à região orbito-medial, teria a função de inibir comportamentos inadequados ou prejudiciais, relacionados a uma resposta emocional reflexiva, do tipo irritabilidade ou impulsividade, no que constituiria o chamado "controle inibitório de interferência”. Já a região dorso-lateral do córtex pré-frontal relacionar-se-ia a concentração, planejamento e memória recente. Sua disfunção, a chamada "disfunção cognitiva executiva", facilitaria o aparecimento de comportamentos agressivos.

Certamente, deve-se ter cautela ao aplicar os achados obtidos para se inferir presença de lesão cerebral nesses indivíduos. Porém, avolumam-se as evidências no sentido de que 
esses indivíduos (homicidas) necessitam de uma avaliação mais individualizada, em nível psiquiátrico, a exemplo do que se faz em outros países. ${ }^{17}$ Ao mesmo tempo, a pesquisa em psiquiatria forense necessita de uma melhor depuração das populações estudadas. No estágio atual, considera-se que instrumentos como o HARE PCL-R, por seu poder em predizer violência e recidividade, são imprescindíveis para o estabelecimento de subgrupos de indivíduos violentos, de forma a permitir tanto um maior aprofundamento da pesquisa, quanto um manejo mais adequado desses indivíduos por parte de quem tem, para tal, atribuição da sociedade, ou seja, o Sistema Jurídico-Criminal e Penal.

Os estudos com populações violentas freqüentemente se res-

\section{Referências}

1. Cherkasky S, Hollander E. Neuropsychiatric aspects of impulsivity and aggression. In: Yudofsky SC, Hales RE, editors. Washington (DC): Am Psychiatric Press; 1997. p. 485-99.

2. Blake PY, Pincus JM, Buckner C. Neurologic abnormalities in murdererers. Neurology 1995;45:1641-7.

3. Bus KG, Zagar R, Hughes JR, Arbit J, Bussel RE. Adolescents who kill. J Clin Psychology 1990;46(4):472-85.

4. Heinrichs RW. Frontal cerebral lesions and violent incidents in chronic neuropsychiatric patients. Biol Psychiatry 1989;25:174-8.

5. Krakowski MI, Convit A, Jaeger S. Neuropsychological impairment in violent schizophrenic inpatients. Am J Psychiatry 1989;146(7):849-53.

6. Mark VK, Erwin FR. Violence and the brain. New York: Harper and Row; 1970 .

7. Monroe RB. Brain dysfunction in prisoners. In: Hays JR, Roberts TK, Solway KS, editors. Violence and the violent individual. Lancaster: MTP Press; 1981.

8. Pontius AA, Yudowitz LLB. Frontal Lobe System Dysfunction in some criminal actions as shown in the Narratives test. J Nerv Ment Dis 1980;168(2):111-7.

9. Saver JL, Salloway SP, Devinsky O, Bear DM . Neuropsychiatry of aggression. In: Fogel BJ, Schiffer RB, Rao SM, editors. Neuropsychiatry. Baltimore: Williams \& Wilkins; 1996. p. 523-48.

10. Spellacy F. Neuropsychological differences between violent and nonviolent adolescents. J Clin Psychology 1977:33(4):96-9.

11. Volavka J. Neurobiology of violence. Washington (DC): Am Psychiatric Press; 1995.

12. Volavka J, Martell D, Convit A. Psychobiology of the violent offender. J For Sci 1992;37(1):237-51.

13. Volkow ND, Tancredi L. Neural substrates of violent behavior: a preliminary study with PET. Br J Psychiatry 1987;151:668-73.

14. Yeudall LT, Fromm-Auch D, Davies R. Neuropsychological impairment of persistent delinquency. J Nerv Ment Dis 1982;170(5):257-65.

15. Goldstein M. Brain research and violent behavior. Arch Neurology 1974;30:1-35.

16. Zagar R, Arbit J, Sylvies R, Busch KG, Hughes JR. Homicidal adolescents: a replication. Psychol Rep 1990;67:1235-42.

17. Jozef F. O criminoso homicida - estudo clínico - psiquiátrico [tese]. Rio de Janeiro: COSEGIP; 1998.

18. Hare RD. Manual for the Hare Psychopathy Checklist-Revised. Toronto: Multi-Health Systems; 1991.

19. Lewis DO. Adult antisocial behavior and criminality. In: Kaplan HI, Sadock BJ, editors. Compreensive Textbook of Psychiatry V. Baltimore: Williams \& Wilkins; 1989. p. 1400-5.

20. Lishman WA. Brain damage in relation to psychiatric disability after head injury. Br J Psychiatry 1968;114:343-410. sentem da dificuldade em selecionar adequadamente a população violenta, mercê da dificuldade em definir o comportamento violento a ser estudado, estabelecendo critérios uniformes para toda amostra. Essa é uma importante vantagem do estudo de populações de homicidas. Também os instrumentos de avaliação psicológica tradicional, de auto-relato, como o MMPI, revelaram-se estéreis quando empregados com populações forenses, por não discriminarem entre populações sabidamente diversas. Assim, na pesquisa empírica em psiquiatria forense, especialmente no estudo de populações violentas, considerase como recomendável o amplo emprego de instrumentos como o PCL-R, assim como a testagem neuropsicológica, privilegiando-se o subteste Mosaico.

21. Sreenivasan S, Kirkish P, Eth S, Mintz J, Hwang S, Van Gorp W et al. Predictors of recidivistic violence in criminally insane and civilly commited psychiatric inpatients. Int J Law Psychiatry 1997;20(2):279-91.

22. Hare RD. Psychopathy: A clinical construct whose time has come. Crim Justice Behavior 1996;23:25-54

23. Cleckley HM. The mask of sanity. St. Louis: Mosby; 1976.

24. Jozef F, Silva JAR. Psicopatia e alterações frontais em homicidas. J Bras Psiquiatria 1999;48(1):29-34.

25. Silva JAR. Criminalidade e distúrbio mental [tese]. Rio de Janeiro: COSEGIP; 1981.

26. Cunha JA. Escalas Wechsler. In: Psicodiagnóstico. Porto Alegre: Artes Médicas; 1993.

27. Lezak MD. Neuropsychological Assessment. New York: Oxford University Press; 1995.

28. Serin RE, Amos NL. The Role of Psychopathy in the assessment of dangerousness. Int J Law Psychiatry 1995;18(2):231-8.

29. Rice ME, Harris GT. Psychopathy, schizophrenia, alcohol abuseand violent recidivism. Int J Law Psychiatry 1995;18(3):333-42.

30. Organização Mundial da Saúde. Classificação de transtornos mentais e de comportamento (CID 10): Descrições clínicas e diretrizes diagnósticas. Porto Alegre: Artes Médicas; 1993.

31. Hare RD. Comparison of procedures for the assessment of psychopathy. J Cons Clin Psychology 1985;53(1):7-16.

32. Hart SD, Forth AE, Hare RD. Performance of criminal Psychopaths on selected neuropsychological tests. J Abn Psychology 1990;99(4):374-9.

33. Cornel DG, Warren J, Hawk G, Stafford E, Oram G, Pine G et al. Psychopathy in instrumental and reactive violent offenders. J Cons Clin Psychol 1996;64(4):783-90.

34. Mega MS, Cummings JL. Frontal-subcortical circuits and neuropsychiatric disorders. J Neuro-psychiatr Clin Neurosc 1994;6:358-70.

\section{Correspondência}

Flavio Jozef

Serviço de Psiquiatra Forense

Instituto de Psiquiatria da UFRJ

Av. Venceslau Braz, 76 - fundos

22.290-140 Botafogo, Rio de Janeiro, RJ

Tel.: (0xx21) 265-5957

Fax: (0xx21) 557-2928

E-mail: flaviojozef@rj.sol.com.br 